\title{
Association Between Suicide and Drinking Habits in Adolescents
}

\author{
Ji Won Lee ${ }^{1}$, Bong-Jo Kim ${ }^{1,2}$, Cheol-Soon Lee ${ }^{2,3}$, Boseok Cha ${ }^{1,2}$, So-Jin Lee ${ }^{1,2}$, Dongyun Lee ${ }^{2,3}$, \\ Jiyeong Seo ${ }^{3}$, Young-Ji Lee ${ }^{3}$, Youn-Jung Lee ${ }^{1}$, Eunji Lim ${ }^{3}$, and Jae-Won Choi ${ }^{1}$ \\ ${ }^{1}$ Department of Psychiatry, Gyeongsang National University Hospital, Jinju, Korea \\ ${ }^{2}$ Department of Psychiatry, Gyeongsang National University College of Medicine, Jinju, Korea \\ ${ }^{3}$ Department of Psychiatry, Gyeongsang National University Changwon Hospital, Changwon, Korea
}

Objectives: Adolescent suicide is a serious social problem. Adolescent alcohol use is one of the most important risk factors for adolescent suicide. This study aimed to identify the relationship between drinking habits and suicide among Korean adolescents.

Methods: Data from the 14th and 15th Korean Youth Risk Behavior Web-based Survey, conducted in 2018 and 2019, were used for analysis. Multiple logistic regression analysis was used to identify the relationship between drinking habits_including the age of drinking initiation, frequency of drinking, average drinking amount, frequency of drunkenness-and suicidal ideation, plans, and attempts.

Results: Even after adjusting for age, sex, school grade, academic achievement, socioeconomic status, depression, stress, and drinking habits, the frequencies of drinking and drunkenness increased the risk of suicide attempts. Suicide attempts were associated with the frequency of drinking in girls and middle school students, and with the frequency of drunkenness in boys and high school students.

Conclusion: This study identified associations between drinking habits (the age of drinking initiation, frequency of drinking, average amount of drinking, frequency of drunkenness) and suicidal behavior in adolescents. Our findings suggest that to prevent adolescent suicide, it might be necessary to investigate drinking habits, including the frequencies of drinking and drunkenness. Moreover, considering the differences in sex and school grade, it is important to include the individual group characteristics when evaluating drinking habits.

Keywords: Suicide; Adolescent; Alcohol drinking.

Received: July 27, 2021 / Revision: August 31, 2021 / Accepted: September 2, 2021

Address for correspondence: Jae-Won Choi, Department of Psychiatry, Gyeongsang National University Hospital, 79 Gangnam-ro, Jinju 52727, Korea Tel: +82-55-750-9788, Fax: +82-55-759-0003, E-mail: lingker77@naver.com

\section{INTRODUCTION}

Since 2011, suicide has been the leading cause of death among the youth (age between 9 and 24 years) in Korea [1]. According to the 2019 Annual Report on the Causes of Death Statistics, 5.9 per 100000 adolescents aged between 10 and 19 years committed suicide, $2.7 \%$ more than the previous year [2]. Adolescent suicide, aside from being a personal tragedy, has a serious impact on peers and family [3], and can also cause serious social costs since it leads to the loss of future productivity and economic values [4]. In 2015, the Korea Institute for Health Insurance Policy Research reported in "An Estimation of Economic Costs of 5 Major Diseases" that the costs of direct and indirect societal loss from adolescent suicide reached 388.5 billion KRW [5].

Given their incomplete brain development and lack of emotional control, adolescents have a poorer situational judgment

This is an Open Access article distributed under the terms of the Creative Commons Attribution Non-Commercial License (https://creativecommons.org/licenses/by-nc/4.0) which permits unrestricted non-commercial use, distribution, and reproduction in any medium, provided the original work is properly cited. and are more impulsive than adults [6]. Impulsivity is reported to be a risk factor for suicide $[7,8]$; hence, the risk of adolescent suicide can further increase because of it. Considering that a suicide attempt in itself may leave serious sequelae and that it has a negative impact not only on the individual but also the family and peers, it is essential to recognize the risk factors for suicide and intervene early.

Previous studies have reported that suicide occurs due to a series of factors, including mental, social, cultural, biologi$\mathrm{cal}$, and environmental factors, rather than a single one $[9,10]$. Alcohol and drug use have been identified as major causes of suicide [10]. The American Academy of Child and Adolescent Psychiatry (AACAP) and the American Psychiatric Association assessed alcohol and drug abuse as one of the greatest risk factors for adolescent suicide [11,12]. Further, the AACAP recommended that all adolescents presenting suicidal ideation and/or behavior should be tested for substance use, including alcohol and drugs [11,13].

In particular, alcohol use is reportedly more strongly associated with adolescent suicidal behavior than the use of other 
drugs [14]. Drinking itself increases psychological distress and impulsivity and reduces the problem-solving capacity, thus increasing the risk of suicide attempts [15]. Furthermore, drinking causes disinhibited behavior, which may not only facilitate suicidal ideation but also lead to impulsive suicide attempts [16]. Previous studies conducted in and outside of Korea reported that the suicidal ideation risk increased in groups with drinking experience compared to groups without $[17,18]$. Studies investigating the association between drinking habits (such as the amount of drinking and age of drinking initiation) and suicidal behavior found that higher alcohol consumption increased the risk of adolescents attempting suicide $[11,19]$ and that the earlier the age of drinking initiation, the higher the suicide attempt risk [20-22]. These findings suggest the need to assess not only drinking status but also various drinking habits to prevent suicide among adolescents. However, to date, research on drinking habits and their associations with suicide in large-scale studies on adolescent populations has been inadequate.

Suicidal ideation, suicide attempts, and death by suicide are on the same continuum [22]. Experiences of serious suicidal ideation and suicide plans and/or attempts increase the likelihood of death by suicide. Accordingly, it is important to classify in detail the risk factors of suicidal ideation, plans, and attempts, and accurately determine the risk level [23]. Regarding the association between drinking habits and suicide, it is necessary to assess not only the drinking status but also various other drinking habits. This study focused on the frequencies of drinking and drunkenness, in addition to the age of drinking initiation and the amount of drinking.

We hypothesized that the suicide risk would increase with the frequency of drinking and drunkenness. This study aimed to investigate the impact of each drinking habit on suicidal ideation, plans, and attempts by adjusting the effects of all other drinking habits examined in the survey.

\section{METHODS}

\section{Study subjects}

The 2018 and 2019 data of the Korea Youth Risk Behavior Web-based Survey were analyzed, which included the frequency of drunkenness. The survey is an annual web-based survey conducted since 2005 by the Korea Ministry of Health and Welfare, Center for Disease Control and Prevention, and Ministry of Education to examine the status of health behaviors in Korean youth from the first year of middle school to the third year of high school. The questionnaires were selfadministered anonymously in school computer labs by students who consented to participate in the online survey. The survey sample was designed using the stratified sampling method. A total of 60040 and 57303 students across 800 schools participated in the 14th and 15th edition of the survey, respectively. The current study was approved by the Institutional Review Board of Gyeongsang National University Hospital Institutional Review Board (IRB approval number: GNUH 2021-07-028).

\section{Analysis items}

\section{Sociodemographic characteristics}

Sociodemographic characteristics included sex, age, socioeconomic status, and academic achievement. School grades were grouped into two categories: middle school and high school. Economic status and academic achievement were grouped into five categories: upper, upper middle, middle, lower middle, and lower.

\section{Drinking habits}

For the variables pertaining to drinking habits, the age of drinking initiation, frequency and amount of drinking, and frequency of drunkenness were considered. Regarding the age at which respondents had one or more glasses of alcohol for the first time (not taking into account occasions such as ancestral rites on death anniversaries, traditional holidays or religious rites), the 13 age categories used to code the original data were re-classified into 3 categories as follows: "pre-teen (pre-school to 6th grade)," "middle teen (7th to 9th grade)," and "late teen (10th to 12th grade)." The amount and frequency of drinking and frequency of drunkenness were assessed using a timeframe of the last 30 days. The question regarding the frequency of drinking was "in the last 30 days, on how many days did you have one or more glasses of alcohol?"; the answer options included the following 7 categories: "none," "1-2 days per month," "3-5 days per month," "6-9 days per month," "10-19 days per month," "20-29 days per month," and "daily." The questions regarding the amount of drinking and the frequency of drunkenness were asked only to the adolescents who responded that they had at least one glass of alcohol in the last 30 days. The average amount of drinking in the last 30 days was assessed using the following 5 categories: "1-2 glasses of Soju (less than one bottle of beer)," "3-4 glasses of Soju (2 bottles of beers, 3 glasses of wine)," "5-6 glasses of Soju (3 bottles of beers, 5 glasses of wine)," "1-2 bottles of Soju ( 4 bottles of beers, 6 glasses of wine), and " 2 or more bottles of Soju ( 8 bottles of beers, 12 glasses of wine)." Lastly, the frequency of drunkenness was assessed through a question regarding the number of days in the last 30 days when respondents drank so much they lost their consciousness or memory; the answer categories included "none in the last 30 days," "1-2 days per month," "3-4 days per month," and 
“5 or more days per month."

\section{Mental health factors}

The questions regarding suicidal ideation and suicide plans and attempts assessed the respective experiences in the last 12 months, with the answer options of "yes" and "no." The question regarding depression inquired whether or not, in the last 12 months, respondents felt sadness or despair consecutively for two weeks to the extent that they could not carry on daily activities, the answer options being "yes" and "no." Regarding stress, the question, "How stressed do you usually feel?," was asked with the following 5 answer categories: "extremely stressed out," "very stressed out," "somewhat stressed out," "little stressed out," and "not at all stressed out."

\section{Statistical analysis}

Complex sample analysis was performed after stratification, and the cluster and weight variables were specified. Crosstab analysis was conducted according to the presence or absence of suicidal ideation, plans, and attempts, with the aim of examining differences in sex, age, school grade, economic status, academic achievement, stress, and depression. Logistic regression analysis was conducted to assess the relationships between drinking habits and suicidal ideation, plans, and attempts. The age of drinking initiation, frequency and amount of drinking, and frequency of drunkenness were considered dependent variables, and suicidal ideation, plans, and attempts, independent variables. Age, sex, school grade, academic achievement, socioeconomic status, depression, and stress, as well as drinking habits that might influence one another (the frequencies of drinking and drunkenness, average amount of drinking) were used as covariates. One-way analysis of variance (one-way ANOVA) and post-hoc analysis were conducted to examine differences in drinking habits according to the presence or absence of suicidal ideation, plans, and attempts. All statistical analyses were performed using IBM Statistical Package for Social Science (SPSS) for Windows, Version 25 (IBM Corp., Armonk, NY, USA). The level of statistical significance was set at a p-value $<0.05$.

\section{RESULTS}

\section{Comparison of sociodemographic characteristics}

The number of respondents was 60040, in 2018, and 57303, in 2019. Suicidal ideation was reported by 7976 (13.3\%) and 7498 (13.1\%) respondents in 2018, and 2019, respectively. In 2018, 2631 adolescents (4.4\%) responded that they had made suicide plans, and the corresponding number from 2019 was 2306 (4.0\%). In 2018, 1873 (3.1\%) of subjects responded that they had attempted suicide, while the corresponding num- ber in 2019 reached 1731 (3.0\%). Among males, 635 (2.2\%) and 566 (1.9\%) suicide attempts have been reported in 2018 and 2019, respectively. Among females, attempted suicide has been reported by 1238 (4.1\%) respondents in 2018 and by 1165 (4.0\%) respondents in 2019. Regarding school grade, 1070 (3.6\%) and 1073 (3.6\%) middle school students reported suicide attempts in 2018 and 2019, respectively; the corresponding numbers among high school students were 803 (2.6\%) and $658(2.4 \%)$ in 2018 and 2019, respectively $(\mathrm{p}<0.001)$ (Table 1).

The socioeconomic status was lower in both 2018 and 2019, hence the proportion of adolescents reporting suicidal ideation, plans, and attempts increased $(\mathrm{p}<0.001)$. Furthermore, the proportion of adolescents with suicidal ideation, plans, and attempts increased $(\mathrm{p}<0.001)$ as academic achievement decreased. Regarding stress levels, in both years, the proportions of reported suicidal ideation, plans, and attempts were higher in those who claimed that they were "very stressed out" or "extremely stressed out" than in those who were "little stressed out" ( $\mathrm{p}<0.001)$. In addition, in both years, adolescents experiencing depression also reported higher frequencies of suicidal ideation, plans, and attempts $(\mathrm{p}<0.001)$ (Table 1).

\section{Comparisons of suicidal ideation, plans, and attempts according to drinking habits}

\section{Comparisons of suicidal ideation, plans, and attempts according to the age of drinking initiation}

In both 2018 and 2019, the proportions of adolescents experiencing suicidal ideation, plans, and attempts were higher as the age of drinking initiation was lower $(\mathrm{p}<0.001)$ (Fig. 1A). Post-hoc analysis showed statistically significant differences in all between-group comparisons ( $\mathrm{p}<0.05)$, except when comparing the respondents with no drinking experience to those who began drinking in high school (in the 2019 data). Logistic regression analysis showed that in both years, the earlier the age of drinking initiation, the higher the risk of suicidal ideation, plans, and attempts $(\mathrm{p}<0.001)$ (Table 2$)$. These associations were statistically significant even after adjusting for covariates influencing suicidal experience ( $\mathrm{p}<$ 0.001 ) (Table 2). In particular, the risk of suicide attempt increased in adolescents who began drinking before the 6th grade in elementary school compared to those who started drinking in high school, by 1.4 times in 2018 (adjusted odds ratio $[\mathrm{OR}]=1.421, \mathrm{p}=0.001,[1.124-1.797])$ and by more than two times in 2019 (adjusted OR=2.060, $\mathrm{p}=0.001,[1.578-2.689]$ ) (Table 2).

\section{Comparisons of suicidal ideation, plans, and attempts according to the frequency of drinking}

The proportion of adolescents reporting suicidal ideation, 
Table 1. General demographic, socioeconomic, and mental health factors in Korean adolescents

\begin{tabular}{|c|c|c|c|c|c|c|}
\hline \multirow{3}{*}{ Variables } & \multicolumn{2}{|c|}{ Suicidal ideation } & \multicolumn{2}{|c|}{ Suicide plans } & \multicolumn{2}{|c|}{ Suicide attempts } \\
\hline & 2018 & 2019 & 2018 & 2019 & 2018 & 2019 \\
\hline & $(n=7976,13.3 \%)$ & $(n=7498,13.1 \%)$ & $(n=2631,4.4 \%)$ & $(n=2306,4.0 \%)$ & $(n=1873,3.1 \%)$ & $(n=1731,3.0 \%)$ \\
\hline \multicolumn{7}{|l|}{ Demographic factor } \\
\hline \multicolumn{7}{|l|}{ Sex } \\
\hline Male & $2824(9.6)$ & $2731(9.4)$ & $980(3.3)$ & $904(3.1)$ & $635(2.2)$ & $566(1.9)$ \\
\hline Female & $5152(17.4)$ & $4767(17.1)$ & $1651(5.6)$ & $1402(5.0)$ & $1238(4.1)$ & $1165(4.0)$ \\
\hline Age & $15.14 \pm 0.035$ & $14.99 \pm 0.035$ & $15.01 \pm 0.047$ & $14.84 \pm 0.044$ & $14.92 \pm 0.059$ & $14.72 \pm 0.053$ \\
\hline \multicolumn{7}{|l|}{ School grade } \\
\hline Middle school students & $4142(13.8)$ & $4099(14.1)$ & $1467(5.0)$ & $1349(4.6)$ & $1070(3.6)$ & $1073(3.6)$ \\
\hline High school students & $3834(12.9)$ & $3399(12.2)$ & $1164(3.9)$ & $957(3.4)$ & $803(2.6)$ & $658(2.4)$ \\
\hline \multicolumn{7}{|l|}{ Academic achievement } \\
\hline Upper & $939(12.0)$ & $864(11.4)$ & $355(4.4)$ & $320(4.3)$ & $231(2.9)$ & $210(2.9)$ \\
\hline Upper middle & $1773(11.5)$ & $1622(11.4)$ & $544(3.5)$ & $466(3.2)$ & $348(2.3)$ & $310(2.1)$ \\
\hline Middle & $2092(11.9)$ & $2039(11.9)$ & $661(3.7)$ & $570(3.3)$ & $442(2.5)$ & $443(2.5)$ \\
\hline Lower middle & $2038(15.4)$ & $1852(14.7)$ & $635(4.8)$ & $543(4.2)$ & $500(3.6)$ & $430(3.4)$ \\
\hline Lower & $1134(19.4)$ & $1121(19.8)$ & $436(7.7)$ & $407(7.1)$ & $352(6.1)$ & $338(5.8)$ \\
\hline \multicolumn{7}{|l|}{ Social economic status } \\
\hline Upper & $771(12.3)$ & $708(11.2)$ & $333(5.3)$ & $273(4.4)$ & $215(3.4)$ & $203(3.2)$ \\
\hline Upper middle & 2007 (11.5) & $1831(11.4)$ & $648(3.6)$ & $523(3.1)$ & $408(2.3)$ & $372(2.3)$ \\
\hline Middle & $3446(12.4)$ & $3310(12.1)$ & $1004(3.7)$ & $933(3.4)$ & $757(2.7)$ & $676(2.4)$ \\
\hline Lower middle & $1360(20.5)$ & $1266(20.8)$ & $458(6.8)$ & $405(6.6)$ & $351(5.1)$ & $337(5.4)$ \\
\hline Lower & $392(27.6)$ & $383(30.2)$ & $188(13.3)$ & $172(13.9)$ & $142(9.7)$ & $143(10.6)$ \\
\hline \multicolumn{7}{|l|}{ Mental health factors } \\
\hline \multicolumn{7}{|l|}{ Perceived stress } \\
\hline Extremely stressed out & 3001 (43.3) & $2759(40.9)$ & $1164(16.5)$ & $1030(15.1)$ & $816(11.4)$ & $791(11.4)$ \\
\hline Very stressed out & $3365(19.2)$ & $3151(19.5)$ & $900(5.2)$ & $766(4.7)$ & $647(3.7)$ & $589(3.5)$ \\
\hline Somewhat stressed out & $1344(5.6)$ & $1339(5.8)$ & $409(1.7)$ & $371(1.6)$ & $291(1.2)$ & $270(1.2)$ \\
\hline Little stressed out & $190(2.2)$ & $179(2.1)$ & $98(1.2)$ & $75(0.8)$ & $71(0.8)$ & $43(0.5)$ \\
\hline Not at all stressed out & $76(3.7)$ & $70(3.3)$ & $60(2.9)$ & $64(3.0)$ & $48(2.2)$ & $38(1.6)$ \\
\hline \multicolumn{7}{|l|}{ Depressive mood } \\
\hline No & $1808(4.1)$ & $2030(5.0)$ & $473(1.1)$ & $541(1.3)$ & $332(0.7)$ & $358(0.8)$ \\
\hline Yes & $6168(38.2)$ & $5468(33.9)$ & $2158(13.3)$ & $1765(10.9)$ & $1541(9.4)$ & $1373(8.3)$ \\
\hline
\end{tabular}

plans, and attempts increased with the drinking frequency in both 2018 and 2019 ( $\mathrm{p}<0.001)$ (Fig. 1B). Post-hoc analysis revealed higher proportions of suicidal ideation, plans, and attempts in the group who drank daily showed compared to the other ones $(\mathrm{p}<0.05)$. Logistic regression analysis showed that in both years, the risks of suicidal ideation, plans, and attempts increased with the frequency of drinking $(\mathrm{p}<0.001)$ (Table 2). These associations remained statistically significant after adjusting for the covariates influencing suicidal experience as well as the average amount of drinking and the frequency of drunkenness $(\mathrm{p}<0.001)$ (Table 2). The logistic regression analyses conducted separately for boys and girls revealed a statistically significant association between the frequency of drinking and suicide attempts in girls $(\mathrm{f}=10.897$, $\mathrm{p}<$
0.001 , in 2018; $\mathrm{f}=7.325, \mathrm{p}<0.001$, in 2019), but not in boys. The logistic regression analyses conducted separately for middle school and high school students showed that frequency of drinking was significantly associated with suicide attempts in middle school students ( $\mathrm{f}=5.179, \mathrm{p}<0.001$, in 2018; $\mathrm{f}=5.315$, $\mathrm{p}<0.001$, in 2019), but not in high school students.

\section{Comparisons of suicidal ideation, plan, and attempt according to the amount of drinking}

The proportions of adolescents experiencing suicidal ideation, plans, and attempts increased with the amount of drinking in both 2018 and 2019 (p<0.001) (Fig. 1C). Post-hoc analysis showed higher frequencies of suicidal ideation, plans, and attempts in the groups reporting an average amount of 


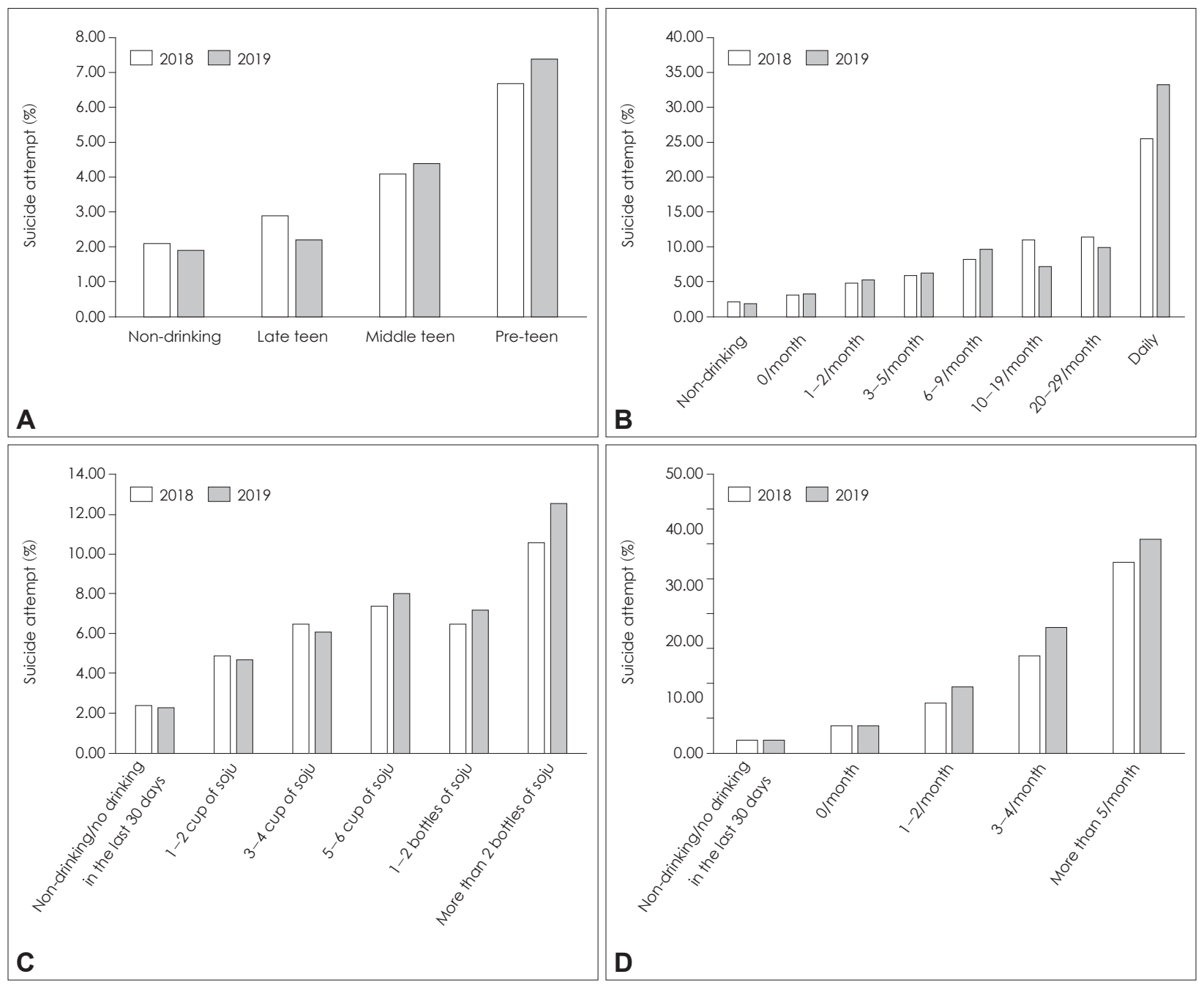

Fig. 1. Changes in suicide attempt rate according to drinking habits. A: Age of alcohol initiation with suidide attempts. B: Frequency of drink with suicide attempts. C: Amount of drink with suicide attempts. D: Frequency of drunkenness with suicide attempts.

drinking of 1-2 glasses, 3-4 glasses, 5-6 glasses, 1-2 bottles, or 2 or more bottles of Soju than in the group reporting to have never consumed alcohol or to have not drunk in the last 30 days $(\mathrm{p}<0.05)$. Logistic regression analysis showed higher risks of suicidal ideation, plans, and attempts with increasing amounts of drinking in both years $(\mathrm{p}<0.001)$ (Table 2$)$. However, the associations between the average amount of drinking and suicidal experience did not remain statistically significant after adjusting for the covariates influencing suicidal experience and frequencies of drinking and drunkenness ( $p>$ 0.05) (Table 2).

\section{Comparisons of suicidal ideation, plans, and attempts according to the frequency of drunkenness}

The proportions of adolescents reporting suicidal ideation, plans, and attempts increased with the frequency of drunkenness in both 2018 and 2019 (p<0.001) (Fig. 1D). Post-hoc analysis revealed significant differences in all between-group comparisons $(\mathrm{p}<0.05)$, except when comparing a drunkenness frequency of 1-2 to 3-4 times per month and 3-4 to 5 or more times per month (in the 2019 data). According to the logistic regression analysis, in both years, the risks of suicidal ideation, plans, and attempts increased with the frequency of drunkenness $(\mathrm{p}<0.001)$ (Table 2). These associations remained significant after adjusting for the covariates influencing suicidal experience, frequency of drinking and average amount of drinking $(\mathrm{p}<0.001)$ (Table 2). The logistic regression analyses conducted separately for boys and girls revealed a statistically significant association between the frequency of drunkenness and suicide attempts in boys $(\mathrm{f}=5.762, \mathrm{p}<0.001$, in 2018; $\mathrm{f}=5.338, \mathrm{p}<0.05$, in 2019), but not in girls. The logistic regression analyses conducted separately for middle school and high school students showed that the frequency of drunkenness was significantly associated with suicide attempts in 


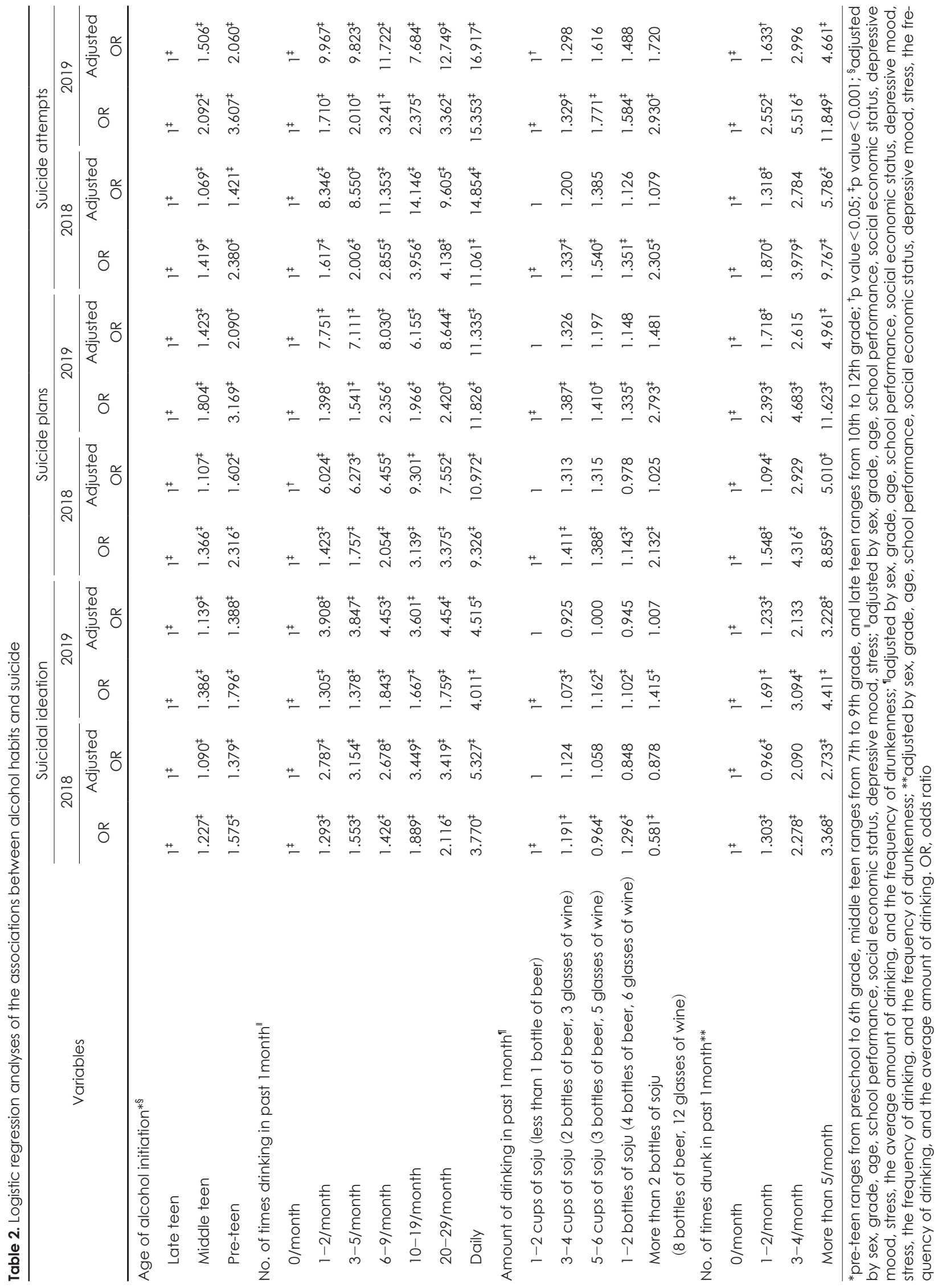


high school students $(\mathrm{f}=9.885, \mathrm{p}<0.001$, in 2018; $\mathrm{f}=6.534$, $\mathrm{p}<$ 0.001 , in 2019), but not in middle school students.

\section{DISCUSSION}

Adolescent suicide is a serious social problem. It is important to assess the risk factors and intervene early to prevent adolescent suicide. Alcohol and drug abuse are considered the greatest risk factors for adolescent suicides [11,13]. Particularly, careful assessment of alcohol use is necessary, since approximately $40 \%$ of Korean adolescents are exposed to drinking according to the Korean Youth Risk Behavior Webbased Survey [24]. This study investigated the associations between drinking habits (such as the age of drinking initiation, frequency and amount of drinking, and frequency of drunkenness) and suicidal ideation, plans, and attempts in adolescents.

An earlier age of drinking initiation was associated with increased proportions of adolescents reporting suicidal ideation, plans, and attempts, as well as with their higher respective risks. This finding supports previous studies emphasizing the dangers of drinking before the age of 13 [21,22], and suggests that the earlier the age of drinking initiation, the higher the risk of suicide attempt. Early adolescence is a period in which identity is not yet established, and individuals are cognitively immature and unstable [19]. Early drinking onset increases the risk of alcohol dependence and alcohol use disorder [25]. Frequent drinking and binge drinking due to alcohol dependence and alcohol use disorder may facilitate suicide attempts [26] and increase the risk of depression or occurrence of negative events [27]. Therefore, the age of drinking initiation should be assessed along with the suicide risk.

The associations between the average amount of drinking and suicidal ideation, plans, and attempts were not statistically significant after adjusting for the covariates influencing suicidal experience, frequency of drinking, and frequency of drunkenness. Previous studies examined the relationship between the amount of drinking and suicide without considering the frequency of drinking, and frequency of drunkenness, in particular $[9,17,19]$. Similarly, in this study the amount of drinking was associated with suicide when not adjusted for the frequencies of drinking and drunkenness. Drinking increases psychological distress, aggression, and impulsivity, and reduces cognitive and problem-solving abilities [3]. However, as the volume of alcohol causing these issues varies among different people, individual differences in drinking habits were not completely adjusted for in the analysis based on the amount of drinking alone. It is believed that how often impulsivity increases due to drinking plays an important role in increasing the suicide risk. The risk of suicide attempts increased by more than 14 times in the 2018 data and by more than 16 times in the 2019 data in the group who drank daily compared to the group who did not drink in the last 30 days, even after adjusting for all drinking habits, including the amount of drinking. Additionally, the risk was increased by more than five times in 2018 and by more than four times in 2019 in the group who experienced drunkenness five times or more in the last 30 days compared to the group without a drunkenness experience in the same timeframe. Although one previous study reported that suicide was associated with the presence or absence of drunkenness in the last 6 months [18], research analyzing the frequency of drinking habits has so far been limited. Hence, follow-up research should be conducted to replicate the current study findings using different subject groups. Nevertheless, the findings of this study suggest the importance of assessing the frequencies of drinking and drunkenness in the suicide risk evaluation.

The analysis conducted separately for boys and girls, aiming to examine the association between drinking habits and suicidal behavior, showed that the average amount of drinking was not associated with suicidal ideation, plans, and attempts in either boys or girls. Regarding drinking habits, the frequencies of drinking and drunkenness were significantly associated with suicidal ideation in both boys and girls. However, the drinking habits associated with suicide plans and attempts were the frequency of drinking, in girls, and the frequency of drunkenness, in boys. Compared to men, women are more vulnerable to neurotoxic changes in the brain due to alcohol, and they show more pronounced impairments in impulse control and cognition and a greater increase in aggression [28]. Due to such vulnerabilities, particularly in women, persistent drinking may increase the likelihood of impulsive suicide attempts by impairing impulse control and enhancing aggression [29]. Accordingly, it is believed that the frequency of drinking per se influences suicide attempts in girls, even without an experience of drunkenness. The analyses of the association between drinking habits and suicidal behavior conducted separately for middle school and high school students revealed that the average amount of drinking was not associated with suicidal ideation, plans, and attempts in either group. The frequencies of drinking and drunkenness were associated with suicidal ideation in both middle school and high school students. However, the drinking habits influencing suicide plans and attempts were the frequency of drinking, in middle school students, and frequency of drunkenness, in high school students. This finding suggests that in middle school students, the risks of suicide plans and attempts increase with the frequency of drinking, even if they may not drink a large amount or to the point at which they subjective- 
ly experience drunkenness.

This study had the following limitations. First, it was a crosssectional study; therefore, it is not possible to confirm causal or temporary relationships between drinking habits and suicidal behavior based on the study findings. Hence, a longitudinal study should be considered in the future to examine the temporal sequence and identify causal relationships. Second, this study did not include a survey on psychiatric history that may impact suicidal behavior among adolescents. Lastly, because drinking habits were self-reported, and no objective assessment was done, the outcome values may have been underestimated or overestimated. Hence, future studies should include the psychiatric history and a 3rd party evaluation of the drinking habits, done by parents and friends.

Despite these limitations, the current study is significant in that it demonstrated associations between individual drinking habits (such as the age of drinking initiation, average amount of drinking, and frequency of drinking and drunkenness) and suicidal behavior by using data from a large-scale sample with a high response rate. In addition, the study is significant in that it evaluated the impact of individual drinking habits on suicidal behavior by adjusting for other drinking habits that may influence one another, as well as the risk factors of suicidal behavior such as academic achievement, socioeconomic status, depression, and stress. The study findings suggest that interventions for the prevention of adolescent suicide should examine not only the drinking status, but also the habits associated with drinking. Students with an early age of drinking initiation, high amount of drinking, or high frequency of drinking or drunkenness may be considered a high suicide risk group and should be carefully observed and proactively responded to. Additionally, based on the found differences between male and female students and between middle school and high school students, it is clear that a more effective approach would be to develop and implement programs for drinking prevention and drinking cessation, differentiated according to sex and school grade (middle school and high school).

\section{CONCLUSION}

The study investigated the associations between drinking habits and suicidal behavior and found that the risk of suicide attempts increased with earlier ages of drinking initiation and higher frequencies of drinking and drunkenness. The findings suggest that, when evaluating the risk of suicide, a detailed assessment of not only the drinking status, but also drinking habits, particularly the frequencies of drinking and drunkenness, is needed. In addition, drinking prevention programs should be developed and implemented by taking into consideration our findings that the frequency of drinking is influential in girls and middle school students, while the frequency of drunkenness has a greater impact in boys and high school students.

\section{Availability of Data and Material}

The datasets generated or analyzed during the current study are available in the Korea Disease Control and Prevention Agency repository (http://www.kdca.go.kr/yhs/).

\section{Conflicts of Interest}

Jae-Won Choi, a contributing editor of the Journal of the Korean Academy of Child and Adolescent Psychiatry, was not involved in the editorial evaluation or decision to publish this article. All remaining authors have declared no conflicts of interest.

\section{Author Contributions}

Conceptualization: Jae-Won Choi. Data curation: Ji Won Lee. Investigation: Youn-Jung Lee, Eunji Lim. Methodology: Ji Won Lee, Jae-Won Choi, Dongyun Lee. Project administration: Bong-Jo Kim, Cheol-Soon Lee. Supervision: Boseok Cha, So-Jin Lee. Validation: Jiyeong Seo, Young-Ji Lee. Writing - original draft: Ji Won Lee, Jae-Won Choi. Writing-review \& editing: Bong-Jo Kim, Cheol-Soon Lee, Boseok Cha, So-Jin Lee, Dongyun Lee, Jiyeong Seo, Young-Ji Lee, Youn-Jung Lee, Eunji Lim.

\section{ORCID iDs}

Ji Won Lee https://orcid.org/0000-0002-0563-6476

Bong-Jo Kim https://orcid.org/0000-0003-2419-7306

Cheol-Soon Lee https://orcid.org/0000-0003-1479-6962

Boseok Cha https://orcid.org/0000-0002-3309-8863

So-Jin Lee https://orcid.org/0000-0003-2904-9206

Dongyun Lee https://orcid.org/0000-0002-3977-3663

Jiyeong Seo https://orcid.org/0000-0002-7329-8296

Young-Ji Lee https://orcid.org/0000-0003-0201-2518

Youn-Jung Lee https://orcid.org/0000-0003-4208-7039

Eunji Lim https://orcid.org/0000-0003-3967-8524

Jae-Won Choi https://orcid.org/0000-0002-4516-1954

\section{Funding Statement}

None

\section{REFERENCES}

1) Statistics Korea. 2020 Statistics on the youth [Internet]. Daejeon: Statistics Korea; [cited 2020 April 27]. Available from: https://kostat.go.kr/portal/korea/kor_nw/1/1/index. board?bmode $=$ read $\& a S e q=381815$.

2) Korean Statistical Information Service (KOSIS). Annual report on the causes of death statistics 2019 [Internet]. Daejeon: Statistics Korea; 2020 [cited 2020 Oct]. Available from: https://kosis.kr/ publication/publicationThema.do?pubcode $=$ YD.

3) Bagge CL, Sher KJ. Adolescent alcohol involvement and suicide attempts: toward the development of a conceptual framework. Clin Psychol Rev 2008;28:1283-1296.

4) Doran CM, Kinchin I. Economic and epidemiological impact of youth suicide in countries with the highest human development index. PLoS One 2020;15:e232940.

5) Hyun KR, Lee SM. Analysis of socioeconomic costs of five major diseases. Health Insur Policy 2014;13:91-107.

6) Reyna VF, Farley F. Risk and rationality in adolescent decision 
making: implications for theory, practice, and public policy. Psychol Sci Public Interest 2006;7:1-44.

7) Kingsbury S, Hawton K, Steinhardt K, James A. Do adolescents who take overdoses have specific psychological characteristics? A comparative study with psychiatric and community controls. J Am Acad Child Adolesc Psychiatry 1999;38:1125-1131.

8) Kashden J, Fremouw WJ, Callahan TS, Franzen MD. Impulsivity in suicidal and nonsuicidal adolescents. J Abnorm Child Psychol 1993;21:339-353.

9) Darvishi N, Farhadi M, Haghtalab T, Poorolajal J. Alcohol-related risk of suicidal ideation, suicide attempt, and completed suicide: a meta-analysis. PLoS One 2015;10:e0126870.

10) Hawton K, Saunders KEA, O'Connor RC. Self-harm and suicide in adolescents. Lancet 2012;379:2373-2382.

11) Aseltine RH Jr, Schilling EA, James A, Glanovsky JL, Jacobs D. Age variability in the association between heavy episodic drinking and adolescent suicide attempts: findings from a large-scale, school-based screening program. J Am Acad Child Adolesc Psychiatry 2009;48:262-270.

12) Govinfo. Suicide prevention and youth: saving lives [Internet]. Washington, DC: United States Senate Committee on Health, Education, Labor and Pensions; 2004 [cited 2004 March 2]. Available from: https://www.govinfo.gov/app/details/CHRG-108shrg92459/ CHRG-108shrg92459.

13) Shaffer D, Pfeffer CR, The Work Group on Quality Issues. Practice parameter for the assessment and treatment of children and adolescents with suicidal behavior. J Am Acad Child Adolesc Psychiatry 2001;40(7 Suppl):24S-51S.

14) McManama O'Brien KH, Becker SJ, Spirito A, Simon V, Prinstein MJ. Differentiating adolescent suicide attempters from ideators: examining the interaction between depression severity and alcohol use. Suicide Life Threat Behav 2014;44:23-33.

15) Sellers CM, Diaz-Valdes Iriarte A, Wyman Battalen A, McManama O'Brien KH. Alcohol and marijuana use as daily predictors of suicide ideation and attempts among adolescents prior to psychiatric hospitalization. Psychiatry Res 2019;273:672-677.

16) Sher L. Alcohol consumption and suicide. QJM 2006;99:57-61.

17) Park HS, Hong SA. Gender comparison of factors affecting the suicide ideation of adolescents - based on the Korea Youth Risk Behavior Web-based Survey, 2019 -. Korean Public Health Res 2020;
46:47-57.

18) King RA, Schwab-Stone M, Flisher AJ, Greenwald S, Kramer RA, Goodman SH, et al. Psychosocial and risk behavior correlates of youth suicide attempts and suicidal ideation. J Am Acad Child Adolesc Psychiatry 2001;40:837-846.

19) Byeon KH, Jee SH, Sull JW, Choi BY, Kimm H. Relationship between binge drinking experience and suicide attempts in Korean adolescents: based on the 2013 Korean Youth Risk Behavior Webbased Survey. Epidemiol Health 2018;40:e2018046.

20) Peltzer K, Pengpid S. Early substance use initiation and suicide ideation and attempts among school-aged adolescents in four Pacific Island countries in Oceania. Int J Environ Res Public Health 2015;12:12291-12303.

21) Swahn MH, Bossarte RM. Gender, early alcohol use, and suicide ideation and attempts: findings from the 2005 Youth Risk Behavior Survey. J Adolesc Health 2007;41:175-181.

22) Swahn MH, Bossarte RM, Ashby JS, Meyers J. Pre-teen alcohol use initiation and suicide attempts among middle and high school students: findings from the 2006 Georgia Student Health Survey. Addict Behav 2010;35:452-458.

23) Lewinsohn PM, Rohde P, Seeley JR. Adolescent suicidal ideation and attempts: prevalence, risk factors, and clinical implications. Clin Psychol (New York) 1996;3:25-46.

24) Korea Disease Control and Prevention Agency, 2019 Korean Youth Risk Behavior Web-based Survey [Internet]. Cheongju: Korea Disease Control and Prevention Agency; [2019 October 30]. Available from: https://www.kdca.go.kr/yhs/.

25) Hingson RW, Heeren T, Winter MR. Age at drinking onset and alcohol dependence: age at onset, duration, and severity. Arch Pediatr Adolesc Med 2006;160:739-746.

26) Bridge JA, Goldstein TR, Brent DA. Adolescent suicide and suicidal behavior. J Child Psychol Psychiatry 2006;47:372-394.

27) Borowsky IW, Ireland M, Resnick MD. Adolescent suicide attempts: risks and protectors. Pediatrics 2001;107:485-493.

28) Agartz I, Momenan R, Rawlings RR, Kerich MJ, Hommer DW. Hippocampal volume in patients with alcohol dependence. Arch Gen Psychiatry 1999;56:356-363.

29) Lee J, Min S, Ahn JS, Park KC, Kim MH, Kim H. Gender differences in the effect of alcohol use on intent and lethality of suicidal attempts. Korean J Psychosom Med 2016;24:236-243. 